HISPANIA NOVA

Revista de Historia Contemporánea

Núm. 19, año 2021

ISSN: 1138-7319 - Depósito legal: M-9472-1998

http://www.uc3m.es/hispanianova

DOSSIER

Tiempos de ocupación: experiencias, rostros y espacios en una Europa en guerra (1936-1945)

\title{
LA ADMINISTRACIÓN BAJO OCUPACIÓN: EVALUACIONES DE UN DILEMA*
}

\begin{abstract}
Administration Under Occupation: Evaluations of a Dilemma.
\end{abstract}
Peter Romijn

University of Amsterdam / NIOD Institue for War, Holocaust and Genocide Studies p.Romijn@,niod.knaw.nl

Recibido: 18-05-2020 - Aceptado: 25-09-2020

Cómo citar este artículo/Citation:

Peter Romijn, "La administración bajo ocupación: evaluaciones de un dilema", Hispania Nova, 19 (2021): 776 a 791

DOI: https://doi.org/10.20318/hn.2021.5897
Copyright: (c) HISPANIA NOVA es una revista debidamente registrada, con ISSN 1138-7319 y Depósito Legal M 9472-1998. Los textos publicados están -si no se indica lo contrario- bajo una licencia Reconocimiento-Sin obras derivadas 3.0 España de Creative Commons. Puede copiarlos, distribuirlos y comunicarlos públicamente siempre que cite su autor y la revista y la institución que los publica y no haga con ellos obras derivadas. La licencia completa se puede consultar en: http://creativecommons.org/licenses/bynd/3.0/es/deed.es
Resumen: Este texto explora la relevancia del estudio de la Administración para el análisis de los regímenes de ocupación en el marco de la II Guerra Mundial. Para ello, el artículo se centra en el estudio de caso de las autoridades locales holandesas, que jugaron un importante papel tanto en las realidades de ocupación y adaptación como en las dinámicas de opresión y colaboración, aparentemente contradictorias. Esta contribución también se ocupa de los parámetros a través de los cuales se ejerció la justicia transicional y se abordó la recuperación de la sociedad holandesa después de la ocupación nazi.

Palabras clave: Ocupación, Países Bajos, Administración local, Adaptación, Colaboración.

\begin{abstract}
This text explores the relevance of the study of the Administration for the analysis of occupation regimes during Second World War. In order to do this, the article focuses on the case study of Dutch local authorities, which played an important role both in the realities of occupation and adaptation and in the apparently contradictory dynamics of oppression and collaboration. This contribution also addresses the parameters through which transitional justice was exercised and the recovery of Dutch society after the Nazi occupation was addressed.
\end{abstract}

Keywords: Occupation, The Netherlands, Local Administration, Accomodation, Collaboration. 


\section{INTRODUCCIÓN: "COMO UN ALCALDE EN TIEMPOS DE GUERRA"1}

"Me siento como un alcalde en tiempos de guerra" es una expresión muy común entre la sociedad neerlandesa actual ${ }^{2}$. Normalmente la usan personas en posiciones de responsabilidad que se ven instadas a explicar por qué tienen que ensuciarse las manos optando por un "mal menor". Por supuesto, el fenómeno no se limita a la situación neerlandesa, ni a tomar medidas opresivas en el ámbito público. No obstante, yo diría que este contexto histórico neerlandés en particular demuestra claramente las presiones implicadas. Suelen ocurrir cuando las personas en puestos de poder sienten la necesidad de legitimar sus acciones apuntando a las consecuencias no deseadas de mantener unos principios aceptados. A este respecto, el discurso subyacente de la responsabilidad suele basarse en pedir la confianza pública sobre la capacidad de las autoridades de producir el mejor resultado posible dadas las circunstancias. Normalmente, ese tipo de actitud es uno de los cimientos de la estructura administrativa en momentos de crisis, no obstante, la legitimidad de los titulares puede erosionarse fácilmente y tambalearse si su desempeño no consigue proteger contra la opresión y la catástrofe ${ }^{3}$. La cuestión general

\footnotetext{
${ }^{1}$ Mi proyecto de investigación 'Mayors in Wartime' está basado en una amplia investigación sobre todo en: 1) los archivos del Ministerio del Interior neerlandés Archivos nacionales, La Haya: Gabinete 18141949; Archivos del personal del gabinete (personeelsdossiers); Zuiveringsarchief (Archivo de los procedimientos de purga); CABR (Archivos centrales de procedimientos de justicia penal especiales); 2) las colecciones del NIOD Institute for War, Holocaust and Genocide Studies, Ámsterdam: Reichskommissariat - Stab; - Präsidialabteilung; - Beauftragten des Reichskommisars; - Abteilung niederländische Personalangelegenheiten; Generalkommissariat für Verwaltung und Justiz-Stab, Hauptabteiling Inneres; Generalkommissariat für das Sicherheitswesen; el Archivo del NSB (Movimiento nacionalsocialista en los Países Bajos); las actas de reuniones de los Secretarios Generales y de los Abogados Generales-Directores en funciones de la Policía; y 3) la abundante literatura relevante.

${ }^{2}$ Sobre los alcaldes en los Países Bajos: Peter Romijn Burgemeesters in Oorlogstijd. Besturen onder Duitse bezetting (Amsterdam: Balans, 2006) y Peter Romijn 'Ambitions and Dilemmas of Local Authorities in the German-Occupied Netherlands, 1940-1945' en: Bruno De Wever, Herman Van Goethem y Nico Wouters (Eds.) Local Government in Occupied Europe (1939-1945) (Gent: Academia Press, 2006) pp. 33-66.

${ }^{3}$ Sobre el enfoque de legitimación en acción: Martin Conway y Peter Romijn (Eds.) The War for Legitimacy in Politics and Culture 1936-1946 (Oxford/Nueva York: Berg, 2008).
} 
se verá ampliamente reflejada en la dinámica de la memoria colectiva sobre cómo las burocracias funcionaron como parte de regímenes opresores.

Casi más de 75 años después, toda alusión en los Países Bajos a la dramática experiencia de la ocupación alemana y del dominio nacionalsocialista continúa generando mucho debate público. Durante la conmemoración de las víctimas del régimen nazi, el rey Willem-Alexander incluso expresó su malestar por la posición que tomó su bisabuela, la reina Wilhelmina, durante la Segunda Guerra Mundial. Desde el exilio en Londres, dio muchos discursos radiofónicos a los Países Bajos ocupados, pero casi ninguno trató de la dificultad de los judíos perseguidos ${ }^{4}$. Otro indicador es la expresión común de sentirse "como un alcalde en tiempos de guerra". Esta frase me inspiró a estudiar la posición altamente ambivalente de las autoridades locales en los Países Bajos durante la Segunda Guerra Mundial. En esta contribución discuto la experiencia de la ocupación nazi en los Países Bajos y las evaluaciones legales y morales que prosiguieron desde la perspectiva de los alcaldes holandeses. No obstante, indicaré que tanto la experiencia como el legado del problema están muy unidos a un fenómeno más general de reconciliarse con los problemas tras la guerra y de repensar la ciudadanía.

¿Por qué son de especial interés los alcaldes a este respecto? La explicación se puede encontrar en la posición dual que tienen bajo la ley constitucional holandesa, una posición seriamente mermada bajo el régimen de ocupación. Los alcaldes lideran el nivel más bajo de gobierno, la municipalidad, claramente son los más cercanos a la población y a sus preocupaciones diarias. Al mismo tiempo, el estado central, tomado por el régimen de ocupación nacionalsocialista, los desplegó como instrumentos de gobierno autoritario, vigilando a la sociedad y llevando a cabo persecuciones racistas. Esa mezcla de tener responsabilidad funcional a favor del bienestar de sus ciudadanos y ser parte de una estructura de estado opresiva conllevó muchos problemas políticos y morales para los alcaldes. Por ello, su dilema relativo al "mal menor" se convirtió en un tropo en la memoria colectiva y las lecciones de la guerra.

\footnotetext{
${ }^{4}$ https://www.royal-house.nl/documents/speeches/2020/05/04/speech-by-king-willem-alexander-nationalremembrance-day-4-may-2020
} 


\section{LA EXPERIENCIA DE LA OCUPACIÓN Y ADAPTACIÓN EXTRANJERA}

En mayo de 1940, las fuerzas armadas alemanas invadieron y conquistaron los Países Bajos. En esos momentos, la población neerlandesa constaba de 9 millones de personas, y unos mil alcaldes, nombrados por el estado central, que lideraban más o menos el mismo número de administraciones municipales, desde pequeños pueblos a ciudades importantes. A pesar de que el ejército neerlandés había sido derrotado y forzado a rendirse, las autoridades locales, incluyendo los alcaldes, permanecieron en sus puestos. Se dedicaron a coordinar las medidas de emergencias, incluyendo evacuaciones de civiles a gran escala, y a desarrollar acuerdos prácticos con los comandantes militares alemanes. Como el gobierno, incluido la Reina, se exiliaron, la burocracia estatal central tardó bastante tiempo en recomponerse. Mientras tanto, los administradores locales tuvieron que ocuparse de la conmoción de la derrota. Bajo unas condiciones muy difíciles consiguieron mantener a la sociedad unida. Por ello, asumieron que tenían que continuar sus funciones bajo la ocupación. Muchos alcaldes impulsaron la recuperación material e inclusive espiritual. Para ese fin, se mostraron listos a cooperar para lidiar con los nuevos mandatarios ${ }^{5}$.

El 29 de mayo de 1940, Adolf Hitler nombró un gobierno civil de ocupación bajo el Reichskommissar Arthur Seyss-Inquart. Al hacerlo, demostró el propósito político principal de la ocupación. El interés del Reich alemán determinó la misión de Seyß-Inquart en los Países Bajos en primer lugar ${ }^{6}$. El nombramiento del Reichskommissar dejó claro en primer lugar que Hitler no había establecido aún el destino a largo plazo del estado neerlandés, ahora definido como "territorios ocupados". En el imperio alemán, ese tipo de funcionario se ocupaba de remangarse las manos y preparar la transición de ese territorio al nuevo orden nacionalsocialista. El plazo y resultado esperado era desconocido, pero, en cualquier caso, Seyß-Inquart se ocupó

\footnotetext{
${ }^{5}$ Werner Warmbrunn, The Dutch under German Occupation (Stanford, SUP, 1963); Gerhard Hirschfeld Nazi rule and Dutch collaboration : the Netherlands under German occupation, 1940-1945 (Oxford: berg, 1988); Peter Romijn 'Managing the integration of the occupied Dutch territories in Hitler's empire', en Peter Eigner, Herbert Matis, Adreas Resch (Hg.), Entrepreneurship in schwierigen Zeiten. Unternehmertum, Karrieren und Umbrüche während der ersten Hälfte des 20. Jahrhunderts (Viena: Lit Verlag, 2007), 309-330.

6 Johannes Koll Arthur Seyß-Inquart und die deutsche Besatzungspolitik in den Niederlanden (19401945) (Viena/Colonia/Weimar: Böhlau, 2015).
} 
formalmente del ejercicio del gobierno nazi en los Países Bajos, en nombre de Adolf Hitler. Desde el inicio, no se mostró preparado para compartir la responsabilidad con las autoridades holandesas nativas. En los Países Bajos, al igual que en otros estados de Europa occidental ocupados, Berlín decidió usar burócratas nativos como instrumentos del gobierno alemán ${ }^{7}$.

Al mismo tiempo, este movimiento implicó una violación de los principios del derecho internacional, por ejemplo, la Convención de 1907 de La Haya requería una administración militar bajo la ocupación. ${ }^{8}$ A pesar de violar tan claramente la legalidad, la dirección de los funcionarios públicos holandeses decidió continuar en sus puestos bajo el control de los nuevos mandatarios. Las expresiones típicas utilizadas eran: "prevención del caos" y "comportamiento responsable" para proteger los intereses de la población en general. De esa forma, esperaban servir el propósito político de evitar la aparición de un nuevo régimen radical bajo el formato del gobierno directo de los nazis neerlandeses ${ }^{9}$. Confiaron en que la falta de legalidad desde la perspectiva del derecho internacional no evitaría la legitimidad más amplia de su posición en el contexto de la ocupación. Lo último dependía también de su capacidad de trabajar en el interés del pueblo neerlandés y el grado de reconocimiento del funcionariado público por parte de los ciudadanos.

A pesar de garantizar lo contrario, Seyss-Inquart organizó su gobierno de forma que politizara la administración holandesa. Formalmente, el Reichskommissar lideraba una "administración de supervisión", pero desde el inicio interfirió hasta en los detalles más pequeños de la administración para dirigirla hacia sus propósitos. Bajo su autoridad, cuatro Generalkommissare se ocuparon de poner a la administración neerlandesa, al igual que a amplios grupos de la sociedad civil, "en línea" con el nuevo régimen. Este proceso se puede denominar "nazificación", es decir, la reorganización de la administración y sociedad en general de acuerdo con las políticas autoritarias y

\footnotetext{
${ }^{7}$ Hans Umbreit 'Zur Organisation der Besatzungsherrschaft' en: Johannes Houwink ten Cate und Gerhard Otto (Hrsg.) Das organisierte Chaos "Ämterdarwinismus" und "Gesinnungsethik" Determinanten nationalsozialistischer Besatzungsherrschaft (Berlin: Metropol, 1999), 46-48.

${ }^{8}$ Romijn, ‘Ambitions and Dilemmas', p. 39.

${ }^{9}$ Como repitió con frecuencia el líder del ministerio del interior neerlandés, Karel J. Frederiks, que lo resumió en sus memorias apologéticas "Op de bres" : Overzicht van werkzaamheden op het Department van Binnenlandse Zaken 1940-1944 (La Haya: van Stockum, 1945).
} 
racistas de la Alemania nazi. Por lo tanto, la administración neerlandesa titular fue utilizada en el proceso de nazificación del estado y la sociedad y, quizás a largo plazo, para preparar la integración de la nación dentro del Tercer Reich. La cuestión colonial era sensible para ambas partes. Las élites holandesas temían que el deseo de los alemanes era apropiarse de las Indias Orientales Neerlandesas, pero los líderes alemanes en La Haya y Berlín nunca fueron explícitos sobre la estrategia a largo plazo en este tema, para evitar tensiones con sus aliados en Tokio ${ }^{10}$.

Durante la etapa inicial de nazificación, los principales burócratas holandeses en el centro de gobierno de La Haya estaban lejos de prever el empuje dinámico hacia la nazificación. Preferían asumir que había un interés neerlandés-alemán compartido de mantener la administración en funcionamiento. Las figuras claves en este sentido eran los funcionarios principales de los ministerios del interior, Karel J. Frederiks, y del de economía, Hans Max Hirschfeld. Temían que los alemanes permitiesen al Partido Nazi Neerlandés, el NSB de Anton Mussert o al Nationaal Socialistische Beweging tomar el poder a todos los niveles. Esos hombres suponían que serían capaces de evitar el peligro "proporcionando" orden interno y estabilidad al ocupante a cambio de permitirles mantener el aparato gubernamental en su sitio. En ese aspecto, una absorción por parte del NSB politizaría la administración y crearía caos y división. La cooperación con las autoridades alemanas permitiría a los titulares tratar directa y pragmáticamente con los nuevos gobernantes y de esa forma tener un "mal menor"11.

Para los nuevos gobernantes, por otro lado, era importante enfatizar la legitimidad de su régimen empleando a las autoridades neerlandesas y creando un nivel de entendimiento con ellos. En particular, durante el primer año de la ocupación, las autoridades neerlandesas estaban en "espera", listos para comenzar de cero. Al igual que en la Francia de Vichy y en el resto de Europa, asumían que la guerra había acabado y que el dominio alemán del continente europeo debía aceptarse de facto ${ }^{12}$. De hecho, durante la segunda parte de 1940, hubo voces que describían el presente de aquel

\footnotetext{
${ }^{10}$ Hirschfeld, Nazi-rule and Dutch collaboration, Ch. 1.

${ }^{11}$ Tal y como se explica en Wichert ten Have 1940 : Verwarring en aanpassing; leven in bezet Nederland (Utrecht: Het Spectrum, 2015).

12 Denis Peschanksi 'Legitimacy/Legitimation/Delegitimation: France in the Dark years, a Textbook Case' en: $C E H 13 / 4$, pp. 410-412.
} 
momento como "posguerra". La vuelta a la normalidad era una motivación fuerte en la sociedad ocupada. Tenían la idea de que una actitud positiva de retomar las labores, reparar el daño de la guerra y mirar hacia el futuro les ganaría el respeto de los nuevos gobernantes. Las autoridades alemanas, por su parte, inicialmente tomaron el pulso para ver hasta donde estaban las élites neerlandesas dispuestas a llegar en el camino de la "auto-nazificación". Por lo tanto, al inicio no daban mucho espacio político a Mussert (líder del NSB). Las primeras tensiones surgieron en el verano de 1940, cuando los nazis neerlandeses demandaron compartir el poder y ocupar puestos en el gobierno neerlandés.

\section{LA EXPERIENCIA DE LA OPRESIÓN Y COLABORACIÓN}

Tras varios meses de ocupación, Seyss-Inquart informó a las autoridades neerlandesas de que esperaba que iniciasen "un camino común con el Reich". Comenzó una primera ola de políticas nacionalsocialistas, incluyendo una serie de medidas contra los ciudadanos judíos. En enero de 1941, se pidió a los alcaldes de echaran a los funcionarios y empleados judíos del servicio publico y que montasen un registro central de judíos que vivían en los Países Bajos. Esos fueron los primeros pasos hacia una implantación relativamente "eficiente" de las medidas anti-judías en los Países Bajos: una acumulación de discriminación, aislamiento y deportación de los judíos de los Países Bajos. Como consecuencia, en febrero de 1941, hubo huelgas políticas en Ámsterdam y ciudades cercanas, que se trataron de protestas espontaneas en respuestas a las medidas y provocaciones anti-judías de los nazis neerlandeses. La policía alemana reaccionó rápidamente y con mano dura para reprimir esos disturbios. Al mismo tiempo, las autoridades alemanas comenzaron una purga política gradual de la administración neerlandesa. ${ }^{13}$ En el curso de la ocupación, una mayoría de las posiciones claves en la administración, incluyendo un amplio número de alcaldes importantes, fueron reemplazados por nazis neerlandeses.

13 Un ejemplo claro es el archivo de bteilung niederländische Personalangelegenheiten de Reichskommissariat (NIOD, AmsterdamCollection 23), que muestra cómo el líder de ese departamento, Ernst Althaus, examinó permanentemente todo el órgano del servicio público neerlandés buscando lastres políticos, desde los funcionarios de alto rango hasta los más bajos. 
En junio del mismo año, mientras Hitler invadía la Unión Soviética, el Reichskommissar anunció públicamente: "quien no esté de mi parte está en mi contra"14. De esa forma, delineó una aceleración de la nazificación. En agosto de 1941, SeyssInquart introdujo el "principio de autoridad" nacionalsocialista en el gobierno local. Todos los funcionarios elegidos y órganos perdían su puesto y los alcaldes quedaban bajo control directo alemán y eran solo responsables de la operación de la administración local. Por ello, los principios constitucionales de responsabilidad compartida entre el alcalde, los concejales y los consejos municipales elegidos eran infringidos. Lo mismo podemos decir de los principios de derecho internacional en lo que se refiere a las ocupaciones, que establecen explícitamente que el derecho de los poderes ocupantes de interferir con la administración nativa se limita a lo necesariamente militar y a la salvaguarda del orden público (Convención de 1907 de La Haya, artículo 43). Lo mismo es cierto para el alcance del control y coacción que las autoridades alemanas comenzaron a ejercer en el territorio ocupado. Utilizaron a los alcaldes de muchas formas: para sancionar las acciones policiales, para el racionamiento, los trabajos forzados. Como cabezas nominales de la policía local, los alcaldes eran moralmente responsables de los arrestos políticos. También se les pedía que posibilitaran todo proceso de discriminación administrativa, espolio, aislamiento, persecución y deportación de los judíos entre la población. Esta era una violación del estado de derecho y del principio de igualdad de todos los ciudadanos muy perturbadora $^{15}$.

Como consecuencia, los gobernantes alemanes pidieron a sus pares neerlandeses que escogiesen entre la espada y la pared: ayudar a implantar las políticas nacionalsocialistas o ser reemplazados por personal del "Nuevo Orden". Como afirma el sociólogo neerlandés Cor Lammers, un régimen estable y "constructivo" de ocupación

\footnotetext{
${ }^{14}$ Discurso de Seyss-Inquart en Ámsterdam, 27 junio 1941, publicado obligatoriamente en los diarios neerlandeses, por ejemplo: Rotterdamsch Nieuwsblad 28 juni 1941: https://www.delpher.nl/nl/kranten/view?coll=ddd\&query $=$ Rijkscommissaris\&cql $\% 5 \mathrm{~B} \% 5 \mathrm{D}=\% 28$ date + gt e $+\% 2227-06-1941 \% 22 \% 29 \& c q 1 \% 5 \mathrm{~B} \% 5 \mathrm{D}=\% 28 \mathrm{date}+$ lte $+\% 2230-06-$ $1941 \% 22 \% 29$ \&redirect=true\&page $=2 \&$ identifier $=$ ddd:011002862:mpeg $21: a 001 \&$ resultsidentifier $=$ ddd: 011002862:mpeg21:a0001.

${ }^{15}$ Peter Romijn 'The War (1940-1945)' en: J.C.H. Blom, R.G. Fuks-Mansfeld y I. Schöffer (Eds.) The History of the Jews in the Netherlands (Oxford/Portland: The Littman Library of Jewish Civilization, 2002), 299-335.
} 
extranjero requiere garantizar la cooperación de las élites nativas. Solo si estos últimos están dispuestos a corresponsabilizarse por el funcionamiento efectivo del régimen de ocupación no tendrá que basarse solo en el ejercicio de puro poder, o terror, y tendrá un grado suficiente de legitimidad para hacer que funcione más o menos sin oposición. Según Lammers, las tres condiciones eran instrumentales para esa convergencia de intereses: 1) las élites nativas deberían estar unidas en su enfoque, y listas para compartir responsabilidad entre ellas; 2) las autoridades de ocupación deberían estar preparadas para dejar los asuntos, y responsabilidad, a esas élites para que puedan funcionar correctamente y para mantener el prestigio cara al público; y 3) las fuerzas de ocupación necesitan dejar claro que la ocupación militar es un mal temporal y menor ${ }^{16}$.

Si ponemos a prueba este esquema en el contexto de la ocupación alemana de los Países Bajos, se puede establecer que esas condiciones previas se cumplieron más o menos durante la primera mitad de la ocupación. Las élites neerlandesas estaban unidas, sobre todo porque las políticas partidistas habían cesado, y el funcionariado a todos los niveles utilizó un discurso de responsabilidad, reconstrucción y unidad nacional. Tal y como ellos lo veían, el enemigo común no eran los alemanes en primer lugar, si no los nazis neerlandeses. Por lo tanto, las élites neerlandesas estaban preparadas para pagar el precio de la cooperación con los nuevos gobernantes de Berlín a cambio de dejar fuera a los nazis neerlandeses. Las autoridades alemanas primero dieron la impresión de querer dejar los asuntos internos neerlandeses en manos de los gobernantes nativos. No obstante, cuando les exigieron aceptar las policías nacionalsocialistas, el malestar se extendió rápidamente. Mientras tanto, la perspectiva de una ocupación temporal casi no se trató. Los alemanes no mostraron señales de abandono, las élites neerlandesas no querían presionarles y consideraban que el asunto se decidiría con el resultado de la guerra europea.

Sin embargo, en 1943 empezaron a cambiar las tornas en relación a esas tres condiciones mencionadas por Lammers. Las élites neerlandesas se vieron obligadas a revisar su política de trabajo dentro del contexto del régimen de ocupación. Como resultado de los reveses alemanes en Stalingrado y el norte de África, la derrota alemana empezó a verse como una perspectiva tangible. Además, a inicios de 1943, la

\footnotetext{
${ }^{16}$ Cornelis J. Lammers 'Occupation regimes Alike and Unlike. British, Dutch and French Patterns of Interorganizational Control of Foreign Territories’ en: Organization Studies Vol. 24, pp. 1177-1202.
} 
nazificación progresiva y la opresión política llevaron a una resistencia abierta. Más específicamente, la introducción de trabajos forzados en Alemania demostró ser el factor independiente más fuerte en el descontento y resistencia. A finales de abril y principios de mayo de 1943, estalló una huelga general en muchas zonas del país. Fieles a la estrategia establecida de evitar el caos, las autoridades locales dejaron que los gobernantes alemanes les usasen para poner fin a la huelga. Las autoridades alemanas que trabajaban a nivel provincial informaron que, por lo general, habían conseguido gestionar a los alcaldes para que tomasen medidas para acaban con las huelgas ${ }^{17}$. Aunque en este caso fueran los alemanes quienes presumieran de ello, hubo una crisis de confianza general sobre la autoridad de los alcaldes, lo que llevó a una reconsideración general de los puestos. Los gobernantes nativos empezaron a perder legitimidad en sus puestos cuando demostraron no poder proteger los intereses de los ciudadanos. Hasta las huelgas, los alcaldes y administradores habían llevado a la sociedad hacia la adaptación y la cooperación. A partir de entonces ocurrió lo contrario: la sociedad llevó a la administración por el camino de la no cooperación y resistencia ${ }^{18}$.

A medida que los ejércitos aliados invadían el continente europeo, las autoridades alemanas en los Países Bajos decidieron silenciosamente abandonar sus esfuerzos de ganarse a los neerlandeses para el nacionalsocialismo. Dejaron de gestionar la sociedad, y se prepararon para la defensa militar del Reich. El interés prioritario alemán en occidente era reforzar la "Fortaleza Europa", incluyendo Holanda, para salvaguardar al Tercer Reich. Obligaron a los alcaldes a contratar ciudadanos locales para construir instalaciones militares, contraviniendo el derecho internacional. Cuando el gobierno neerlandés en el exilio anunció que esas actividades estaban explícitamente prohibidas, muchos de los alcaldes que habían permanecido desde antes de la guerra vieron que la legalidad de sus puestos se derrumbaba. Dejaron sus puestos y se escondieron. Los alcaldes restantes, tanto aquellos del "antiguo orden" como los del "nuevo", tuvieron que lidiar con un número creciente de emergencias, como resultado de las inundaciones masivas y evacuaciones de la población civil de las zonas de batalla.

\footnotetext{
${ }^{17}$ Informe de Beauftragte für die Provinz Südholland to the Reichskommissar, 17 mayo 1943, en: P.J. Bouman De April-Meistakingen van 1943 (La Haya: Martinus Nijhoff, 1950), p. 421 (Anexo 58).

18 Peter Romijn Der lange Krieg der Niederlande. Besatzung, Gewalt und Neuroientierung in den vierziger Jahren (Göttingen: Wallstein, 2017), 68.
} 
A medida que las autoridades alemanas introducían la ley marcial, comenzó un gobierno de terror hasta el último invierno de la ocupación ${ }^{19}$.

\section{LOS SUFRIMIENTOS DE LA JUSTICIA TRANSICIONAL}

La liberación de los Países Bajos por las fuerzas aliadas fue un proceso largo que tuvo lugar entre septiembre de 1944 y mayo de 1945. Esos nueve meses de guerra trajeron consigo una enorme destrucción y una crisis humanitaria en las zonas más densamente pobladas del país. Mientras tanto, la administración local como red y como sistema de gobierno se vino abajo. Muchos alcaldes fueron arrestados o ejecutados por la policía alemana en una última ola de terror, otros abandonaron sus puestos y se escondieron, o fueron retirados por los liberadores y la resistencia interna. En esas circunstancias, aparecieron autoridades provisionales de entre la sociedad civil. Eminencias locales se involucraron en el esfuerzo para contrarrestar la emergencia: profesionales médicos, ministros y sacerdotes, profesores y otros profesionales trabajaron juntos bajo la autoridad de la resistencia, las iglesias o la Cruz Roja. Cuando las autoridades militares aliadas y neerlandesas tomaron el control, retuvieron y utilizaron a esos "nuevos hombres" (y mujeres, de hecho). Así, aparecieron administraciones provisionales no oficiales a nivel local. En esos momentos, la legalidad era menos importante que crear la legitimidad sobre lo que quedaba de la sociedad civil. Esas autoridades locales provisionales estuvieron en el centro de la reconstrucción política y administrativa tras la guerra, tanto de abajo arriba como de arriba abajo. Por ello, esas iniciativas contribuyeron al proceso de "normalización" de la administración y reconstrucción del tejido de la sociedad ${ }^{20}$.

Los continuos conflictos crecientes entre los gobernantes nacionalsocialistas y la sociedad ocupada llevaron a un fuerte deseo de preparar una purga política de la administración. El gobierno en el exilio asumió correctamente que era necesario un

\footnotetext{
${ }^{19}$ Ingrid J.J. de Zwarte de Hongerwinter (Amsterdam: Prometheus, 2019) - traducción inglesa en curso. Este contexto forma parte de la contribución de Ingrid de Zwarte a este mismo dossier. Nota de Alejandro Pérez-Olivares.

${ }^{20}$ Peter Romijn "'Liberators and patriots": Military Interim Rule and the Politics of Transition in the Netherlands, 1944-1945' en: Stefan-Ludwig Hoffmann, Sandrine Kott, Peter Romijn, and Olivier Wieviorka Seeking Peace in the Wake of War: Europe, 1943-1947 (Amsterdam: Amsterdam University Press, 2015), 117-142.
} 
"restablecimiento total de la confianza pública" antes de que los funcionarios pudieran desempeñar su papel en la reconstrucción tras la guerra ${ }^{21}$. El primer asunto era cómo definir la colaboración punible; el segundo, cómo administrar la justicia de transición, y el tercero, cómo dar espacio a la colaboración dentro de la memoria colectiva de la ocupación. El proceso real de purga fue un episodio interminable y polémico en la transición, pues aquellos que habían seguido la política del "mal menor" eran atacados, pero intentaban mantener su postura. Ese asunto envenenó el ambiente político en la inmediata posguerra, hasta tal punto que el primer Primer Ministro tras la guerra, Willem Schermerhorn, lo llamó públicamente "el cáncer de nuestra existencia nacional." ${ }^{22}$ Al final, el tercer tema fue el más amplio, y fue tanto sujeto como objeto del proceso histórico de lidiar con la experiencia de la guerra y la ocupación en el periodo de posguerra. La justicia de transición demostró ser un proceso doloroso para identificar los fallos colectivos. En retrospectiva, también fue la primera etapa de la política de dar significado a los dilemas e la administración bajo el régimen extranjero. Por lo tanto, el dilema de ser "alcalde en tiempos de guerra" se ganó su lugar en la memoria social del conflicto.

La definición de los grados punibles de colaboración fue suficientemente difícil por sí sola. Se creó un conjunto amplio de disposiciones legales para tratar de este tema. Los procedimientos de justicia penal se usaron para castigar a todos los miembros del antiguos partido nazi, los procedimientos de justicia administrativa para purgar, desde su oficina, a los nazis y no nazis por igual, a todos aquellos que habían colaborado con el enemigo y desafiado la legalidad del poder político de antes de la guerra. Como en todo proceso de justicia de transición, los temas de conveniencia política fueron los que determinaron en gran parte el resultado, tanto a nivel de casos individuales, como del tema en general. Los alcaldes y otros funcionarios que habían pertenecido al partido nacionalsocialista fueron simplemente despedidos y llevados presos para ser juzgados por tribunales especiales. Los más problemáticos fueron quienes no eran nazis, pero eran miembros del régimen antes de la guerra y habían permanecido en sus puestos

\footnotetext{
${ }^{21}$ Peter Romijn 'Restoration of Confidence: The purge of local government in the Netherlands as a problem of postwar reconstruction' en: Istvan Deak, Jan T. Gross y T. Judt (Eds.) The politics of retribution in Europe. World War II and its aftermath (Princeton: PUP, 2000), pp. 173-193.

${ }^{22}$ Peter Romijn, Boosaardig Bestuur (Amsterdam: Vossius Pers, 2003), p. 17.
} 
hasta el final de la ocupación. Muchos de ellos habían perdido completamente la legitimidad y la confianza de sus ciudadanos.

El caso individual más importante fue el de Frederiks, el funcionario de mayor rango en el Ministerio del Interior, convertido en la cara de la colaboración administrativa con el régimen alemán. Frederiks fue despedido por el gobierno de posguerra por su fracaso en proporcionar liderazgo en el espíritu de la resistencia. Las condiciones de su sentencia fueron bastante moderadas, pero aún así sentó el precedente para despedir a todos aquellos que habían fracasado en sus funciones. En otras palabras, para establecer la autoridad y legitimidad del régimen de posguerra, era necesario despedir a todos aquellos que representaron la actitud general de cooperación al principio de la ocupación, y que ahora se tildaba de colaboración ${ }^{23}$.

\section{RECUPERACIÓN Y APRENDIZAJE}

Paradójicamente, la transición de 1945 del régimen nacionalsocialista a la recuperación y reconstrucción del nuevo reino independiente de los Países Bajos tuvo que ver tanto con lidiar con la derrota como con una expresión de triunfo. De alguna forma, la victoria y la derrota siguieron entrelazadas durante el verano feliz de 1945. Las razones para ello eran bastante obvias: la victoria venía de la mano de los ejércitos aliados que habían liberado al país del enemigo ocupante. Para asegurarle un puesto a la contribución neerlandesa, la resistencia interna contra el ocupante fue elogiada como una parte instrumental de la derrota del enemigo, tanto moral como política. Era suficientemente fácil blandir la colaboración nazi como antipatriota $\mathrm{y}$, como consecuencia, los nazis fueron excluidos de la sociedad. No obstante, la naturaleza de la "colaboración oficial" también tenía que abordarse. ¿Habían sido solo "las células malvadas de nuestro organismo" (como había bautizado al fenómeno de la colaboración la prensa de la resistencia)? ¿O también había contribuido a la supervivencia de la sociedad? De hecho, cuando la primera ola de entusiasmo se apagó, en otoño de 1945 , voces con autoridad dentro del servicio público se levantaron para defender a personas

\footnotetext{
23 Sobre la persona de Frederiks: Peter Romijn 'Kein Raum für Ambivalenzen: Der Chef der niederländischen inneren Verwaltung K.J. Frederiks' en: Gerhard Hirschfeld und Tobias Jersak (Hg.) Karrieren im National-Sozialismus. Funktionseliten zwischen Mitwirkung und Distanz (Frankfurt/Nueva York: Campus Verlag, 2004) pp. 147-172.
} 
como los alcaldes, que habían permanecido en sus puestos e intentado mantener a la sociedad en funcionamiento ${ }^{24}$.

No obstante, en esa falta de legitimidad se asienta una razón mas importante para esa sensación subyacente de derrota y vergüenza. Por la naturaleza del régimen de ocupación, el trabajo del alcalde se había hecho imposible. La violación gradual del derecho internacional y la radicalización del régimen alemán convirtieron a esos administradores en instrumentos de opresión. De forma optimista, esperaban transformar la derrota de 1940, pero al final habían sido derrotados por mantener sus puestos. Se dijo que su involucración con el régimen de ocupación y opresión había servido para un bien mayor: proteger a los ciudadanos lo más posible, y "Alcalde en tiempos de guerra" se convirtió en el nombre popular para ese tipo de dilemas. Por ello, cuando se enfatizan los propósitos nobles y las muestras de tenacidad y audacia antes de rendirse ante las fuerzas enemigas superiores, el dilema se plantea como un discurso de lidiar con la derrota, más que de proclamar una victoria. El aspecto más urgente de la derrota estaba ligado a las implicaciones para la ciudadanía. Bajo la presión de la opresión nazi radical, los alcaldes y otros administradores nativos tuvieron que lidiar con una transformación casi completa del orden constitucional, del estado de derecho y de los principios humanitarios. La exclusión, persecución y deportación de más de 100.000 ciudadanos judíos, especialmente, sorprendió los cimientos morales de la sociedad y a la larga dieron la victoria a ese saborcillo a derrota ${ }^{25}$.

El castigo y rehabilitación de los autores fue parte de la transición de los primeros años de posguerra, que conllevó la vuelta a la democracia política y al estado de derecho. No obstante, la vergüenza estaba imbuida en un proceso más largo de disputa de la barbarie del régimen nazi y de aprender a lidiar con unas experiencias tan dramáticas de forma constructiva. Esta no es mi observación sobre los Países Bajos, pero está relacionada con lo que el historiador estadounidense Konrad Jarausch dice en su reciente obra After Hitler. Recivilizing Germans, 1945-1995 ${ }^{26}$. Mucho de esto

\footnotetext{
${ }^{24}$ Peter Romijn Snel, streng en rechtvaardig. De afrekening met de 'foute' Nederlanders (Ámsterdam: Olympus, 2002 - 2nd pr.).

${ }^{25}$ Peter Romijn and Erik Schumacher 'Transitional Justice in the Netherlands after World War II' en: Nico Wouters (Ed.), Transitional Justice and memory in Europe (1945-2013), 154-155.

${ }^{26}$ Konrad H. Jarausch After Hitler. Recivilizing Germans, 1945-1995 (Oxford: Oxford UP, 2006).
} 
también se aplica a los Países Bajos, donde la involucración de los administradores nativos y de otras élites con el régimen alemán de ocupación ha sido una preocupación continua desde mediados de los 60. A partir de entonces, una fuerte sensación de fracaso ante la maldad ha contaminado gran parte de la memoria colectiva neerlandesa de la Segunda Guerra Mundial. Por ello, la expresión, "me siento como un alcalde en tiempos de guerra" no se ve como una simple exoneración de culpa, su intención original, sino que se ve como una urgencia de tratar con una evaluación de la posición más problemática durante la opresión. Aquí se puede hacer una conexión entre la reapropiación de la ciudadanía, obtener lecciones políticas y la calidad de la recuperación moral.

\section{BIBLIOGRAFÍA}

Bouman De April-Meistakingen van 1943 (La Haya: Martinus Nijhoff, 1950).

Conway, Martin and Romijn, Peter (Eds.) The War for Legitimacy in Politics and Culture 1936-1946 (Oxford/New York: Berg, 2008)

De Zwarte, Ingrid J.J., De Hongerwinter (Amsterdam: Prometheus, 2019).

Frederiks, Karel J., "Op de bres" : Overzicht van werkzaamheden op het Department van Binnenlandse Zaken 1940-1944 (La Haya: van Stockum, 1945).

Hirschfeld, Gerhard, Nazi rule and Dutch collaboration: the Netherlands under German occupation, 1940-1945 (Oxford: Berg, 1988)

Jarausch, Konrad H., After Hitler. Recivilizing Germans, 1945-1995 (Oxford: Oxford UP, 2006)

Koll, Johannes, Arthur Seyß-Inquart und die deutsche Besatzungspolitik in den Niederlanden (1940-1945) (Wien/Köln/Weimar: Böhlau, 2015).

Lammers, Cornelis J., 'Occupation regimes Alike and Unlike. British, Dutch and French Patterns of Interorganizational Control of Foreign Territories', en : Organization Studies Vol. 24, pp. 1177-1202.

Peschanski, Denis, 'Legitimacy/Legitimation/Delegitimation: France in the Dark years, a Textbook Case', CEH 13/4, 410-412.

Romijn, Peter and Schumacher, Erik 'Transitional Justice in the Netherlands after World War II' in: Nico Wouters (Ed.), Transitional Justice and memory in Europe (1945-2013), (Cambridge, Intersentia, 2014)

Romijn, Peter, 'Ambitions and Dilemmas of Local Authorities in the German-Occupied Netherlands, 1940-1945', in De Wever, Bruno, Van Goethem, Herman and Wouters, Nico (Eds.) Local Government in Occupied Europe (1939-1945) (Gent: Academia Press, 2006), 33-66. 
--, 'Kein Raum für Ambivalenzen: Der Chef der niederländischen inneren Verwaltung K.J. Frederiks', in Hirschfeld, Gerhard und Jersak, Tobias (Hg.) Karrieren im National-Sozialismus. Funktionseliten zwischen Mitwirkung und Distanz (Frankfurt/New York: Campus Verlag, 2004), 147-172

--, 'Managing the integration of the occupied Dutch territories in Hitler's empire', in Eigner, Peter, Matis, Herbert and Resch, Adreas (Hg.) Entrepreneurship in schwierigen Zeiten. Unternehmertum, Karrieren und Umbrüche während der ersten Hälfte des 20. Jahrhunderts (Viena: Lit Verlag, 2007), 309-330.

--, 'Restoration of Confidence: The purge of local government in the Netherlands as a problem of postwar reconstruction' en: Istvan Deak, Jan T. Gross y T. Judt (Eds.) The politics of retribution in Europe. World War II and its aftermath (Princeton: PUP, 2000), 173-193

--, "Liberators and patriots": Military Interim Rule and the Politics of Transition in the Netherlands, 1944-1945', in Hoffmann, Stefan-Ludwig, Kott, Sandrine, Romijn, Peter and Wieviorka, Olivier, Seeking Peace in the Wake of War: Europe, 1943-1947 (Amsterdam: Amsterdam University Press, 2015), 117-142

--, Burgemeesters in Oorlogstijd. Besturen onder Duitse bezetting (Amsterdam: Balans, 2006)

--, Der lange Krieg der Niederlande. Besatzung, Gewalt und Neuroientierung in den vierziger Jahren (Göttingen: Wallstein, 2017)

--, Snel, streng en rechtvaardig. De afrekening met de 'foute' Nederlanders (Amsterdam: Olympus, 2002.

--, 'The War (1940-1945)', in Blom, J.C.H., Fuks-Mansfeld, R.G. and Schöffer, I. (Eds.), The History of the Jews in the Netherlands (Oxford/Portland: The Littman Library of Jewish Civilization, 2002), 299-335.

Ten Have, Wichert, 1940: Verwarring en aanpassing; leven in bezet Netherland (Utrecht: Het Spectrum, 2015).

Umbreit, Hans 'Zur Organisation der Besatzungsherrschaft', in Houwink ten Cate, Johannes und Otto, Gerhard (Hrsg.) Das organisierte Chaos. "Ämterdarwinismus" und "Gesinnungsethik" Determinanten nationalsozialistischer Besatzungsherrschaft (Berlin: Metropol, 1999), 46-48.

Warmbrunn, Werner, The Dutch under German Occupation (Stanford: SUP, 1963) 